THE JOURNAL OF TRANSPORT AND LAND USE http://jtlu.org

VOL. 9 NO. 3 [2016] pp. $127-143$

JTLU

\title{
Pedestrian and transit accessibility on a micro level: Results and challenges
}

\author{
Michael A.B. van Eggermond \\ Future Cities Laboratory, Singapore ETH Centre \\ eggermond@ivt.baug.ethz.ch \\ Alex Erath \\ Future Cities Laboratory, Singapore ETH Centre
}

\begin{abstract}
In this paper, we connect two notions of accessibility that are more often than not considered separately: pedestrian accessibility and transit accessibility. We move away from the notion of zonal accessibility and measure fine-grained accessibility using door-to-door travel times. Two pedestrian networks are compared to a baseline scenario considering Euclidean distances for a large metropolitan area in which each individual building is considered as an activity opportunity. It is shown that pedestrian accessibility to jobs differs when pedestrian distances are approximated with different networks that are more representative of reality. Stop-to-stop public transport travel times are extracted from an agent-based simulation of public transport smart card data. The effect of less-than-optimal connections from transit to the pedestrian network, a local measurement, can be seen when calculating the accessibility to all destinations in the city. We suggest moving away from Euclidean-based distance analyses. Limitations can be found in the data available; the connection of buildings to the network becomes important, as does the inclusion of pedestrian crossings. For an inclusive accessibility measure, it will be necessary to calculate generalized costs for pedestrians and generate different pedestrian networks that reflect the limitations of different user groups.
\end{abstract}

Article History:

Received: December 1, 2013

Received in revised form: September 1, 2014

Accepted: December 19, 2014

Available online: July 10, 2015

\section{Introduction}

Transportation researchers generally refer to accessibility as a measurement of the spatial distribution of activities about a point, adjusted for the ability and the desire of people or firms to overcome this spatial separation or "the potential of opportunities of interaction" (Hansen 1959).

Copyright 2015 Michael A.B. van Eggermond and Alex Erath.

doi: $10.5198 /$ jtlu.2015.677

ISSN: 1938-7849 | Licensed under the Creative Commons Attribution - NonCommercial License 3.0.

The Journal of Transport and Land Use is the official journal of the World Society for Transport and Land Use (WSTLUR) and is published and sponsored by the University of Minnesota Center for Transportation Studies. This paper is also published with sponsorship from WSTLUR and the Institutes of Transportation Studies at the University of California, Davis, and University of California, Berkeley. 
While the concept of accessibility has been known since the 1950s, it has only recently drawn attention from practitioners as an attractive indicator measuring the combined effects of transport policies and urban planning. If accessibility measures are considered in transportation planning, mainly zonal, car-based accessibility measures are considered (Handy and Clifton 2001a).

The usage of the term accessibility in everyday life is clearly less abstract than the notion of accessibility as used in transportation research. Here, accessibility can refer to aspects surrounding mobility, ranging from buildings being accessible by a ramp to low-floor buses, improving access to the bus from the bus stop, and the distance to transit. While this practical definition gives insight to aspects of importance when measuring the door-to-door travel experience, it does not capture the concept of reaching destinations and the travel time required to reach these destinations.

Walking, cycling, and public transport are seen as modes that can reduce the negative externalities caused by the use of the automobile, such as congestion, particle emissions, and noise emissions. Moreover, these modes provide equity by equal access, and active modes such as walking and cycling provide additional health and recreational benefits. The popularity of these modes is dependent on, among other things, the level of accessibility provided as compared to the automobile. However, a clear disparity exists between private and public transport accessibility (e.g., Benenson et al. 2010; Hess 2005; Kawabata 1998; Levinson 1998; Salonen and Toivonen 2013; Shen 1998).

To make walking and transit attractive and viable modes of transport, it is necessary to account for the door-to-door travel experience; everyday usage of accessibility clearly reflects this necessity. In the case of public transport, this can be done by increasing headways, improving reliability, and improving access to transit. Improving pedestrian accessibility and the attractiveness of walking can be accomplished in numerous ways, such as by ensuring that walks are useful (e.g., mixed use), safe, comfortable, and interesting (Speck 2012) and by minimizing the difference between beeline and network distances (Weinstein Agrawal et al. 2008).

Accessibility can be computed in several ways. Not only do different types of accessibility measures exist (e.g., Bhat et al. 2002), but the operationalization of these measures also differs in terms of the level of granularity, the travel time used, and the weighting function. Iacono et al. (2013) argue that difficulties in calculating accessibility for non-motorized transport measures arise from problems with data, the zonal structure of transportation models, and transport networks for describing travel by nonmotorized modes. Due to their nature, travel times for walking, cycling, and public transport trips are hard to derive from traditional zonal models. Public transport operates within a small catchment area surrounding the transit stop; walking and cycling are mainly used for shorter trips that are not well represented in zonal models.

It is possible to measure the walking time to opportunities, including transit stops, in a range of ways. Euclidean and Manhattan distances are often used for their simplicity. More realistic pedestrian distances are calculated by means of network distances over road center lines.

This study contributes to previous work by explicitly comparing the outcomes of accessibility computations when different types of pedestrian networks are used to calculate walking times and to calculate door-to-door travel times for public transport. By following this approach and by approximating walking distances with pedestrian networks, the local urban environment is connected to the larger transport system, thus making it possible to evaluate local measures aimed at improving accessibility and to support local planners and citizens with tools to assess and improve accessibility.

Also, by explicitly outlining the different steps followed to calculate accessibility, we aim to highlight different elements of relevance when calculating high-resolution accessibility indicators and provide input to discussions surrounding accessibility computation.

Prior to presenting results in Section 4, Section 2 will present a brief overview of accessibility measures. Section 3 will discuss the methodology followed in this paper and the available data. Section 5 concludes with remaining challenges for the measurement of combined pedestrian and transit accessibility. 


\section{Measuring accessibility}

Geurs and van Wee (2004) identify four components that are relevant when measuring accessibility: (1) a land-use component, (2) a transportation component, (3) a temporal component, and (4) an individual component. An ideal accessibility measure would take these four components into account. These four components all have in common that they relate to the destination and the ease of reaching those destinations.

Five main types of accessibility measures have emerged in literature, all containing one or more of these four components. Bhat et al. (2002) provide an overview of these five types:

1. Spatial separation: The only dimension used in this measure is the distance; it does not consider attractions. However, the most general of these measure consists of the weighted average of the travel times to all other zones under consideration.

2. Cumulative opportunities: This measure takes into account both the distance and the objective of a trip; a travel time or distance threshold is defined and uses the number of potential activities within that threshold as the accessibility for that spatial unit.

3. Gravity measures: This measure includes an attraction factor as well as a separation factor. While the cumulative opportunities measure uses a discrete measure of time or distance and then counts up attractions, gravity-based measures use a continuous measure that is then used to discount opportunities with increasing time or distance from the origin.

4. Utility measures. Two groups of utility measures exist:

- Generalized cost measures estimate total travel costs to go from an origin to a destination, including all relevant time aspects, out-of-pocket costs, and the comfort quality aspect.

- Logsum measures are based on random utility theory and interpret accessibility as the outcome of a set of transport choices. This is calculated by taking, for an individual $n$, the expected value of the maximum of the utilities $\left(U_{i n}\right)$ over all alternative spatial destinations $i$ in choice set $C$. The utility is determined by taking the logsum of $V_{i n}$. This is a linear function with elements representing factors related to accessibility such as the quality of the attraction and the travel costs associated with reaching that attraction.

5. Time/space measures are founded in the space-time geography of Hägerstrand (1970). He used a three-dimensional prism of the space and time available to an individual for partaking in activities. The motivation behind this approach to accessibility is that individuals have only limited time periods during which to undertake activities. As travel times increase, the size of their prisms shrink.

The first three types of accessibility measures are closely related; they consider a transport component (distance) and a number of opportunities that can be reached. It is possible to further specify these measures to approximate preferences of groups or individuals. For instance, modes and/or opportunity types can be excluded from calculations or opportunities can be further categorized. In addition to a description of the opportunities and distances, gravity measures require an impedance parameter. This parameter is usually estimated on survey data and is mode and trip-purpose dependent; they are, however, generally considered constant across different user groups.

\subsection{Pedestrian accessibility}

Despite a wide range of studies calculating or applying automobile accessibility, much less attention has been paid to the calculation of pedestrian accessibility. Handy and Clifton (2001b) identify a gap between the data needed to describe local accessibility, such as data describing the transport network and the location of opportunities, and data available to planning departments. They propose a dual strategy to overcome this gap: a city-wide strategy using available data and the capabilities of GIS and a neighborhood-specific strategy that asks residents themselves to build a detailed accessibility database as a part of a neighborhood planning process. Iacono et al. (2013) highlight the same issues: problems with data, the zonal structure of transportation models, and transport networks for describing 
travel by non-motorized modes. One recent example to calculate pedestrian accessibility is WalkScore (Walk Score 2014). WalkScore gives full points to amenities within a five-minute walk (or 400 meters) and employs a decay function to more distant amenities; beyond a 30-minute walk, no more points are given. Two versions of WalkScore exist: a version only considering crow-fly distances between origins and destinations and, more recently, a version considering network distances. Previous data limitations are overcome by using point-of-interest databases and online network data. Network distances, however, are road center line distances; crossings and building entrances are not included in the calculation.

Research has recognized the need for more detailed pedestrian networks. Karimi and Kasemsuppakorn (2012) present an extensive review of pedestrian network map generation approaches. A distinction can be made between three main approaches: network buffering, collaborative mapping with GPS traces, and image processing. Of these approaches, network buffering requires the least computation effort and image processing the most. The goal of the reviewed studies is mainly to generate pedestrian maps and not so much to generate routable pedestrian networks suitable for accessibility analysis.

\subsection{Public transport accessibility}

Public transport differs from other modes of personalized transport in the sense that it is bound to transit stops, routes and schedules. In practice and research, different methods for calculating accessibility can be found. The Public Transport Accessibility Levels (PTALS) methodology was developed for the London Borough of Hammersmith and is implemented across London. PTALS measures the accessibility of a point to the transport network. The methodology only considers the access to transit; connections of transit to the remainder of the transit system and subsequently to opportunities is not included in the calculation method. A more or less similar approach is followed in Switzerland by the federal spatial planning department (Bundesamt für Raumentwicklung ARE 2011), which uses Euclidean distances to transit instead of network distances and makes a distinction between different types of transit.

Transit accessibility research has focused on the calculation of more realistic transit travel times by incorporating spatially and temporally different travel speeds, waiting times and transfer times, and transfer waiting times (see Salonen and Toivonen 2013). The availability of transit data, network data, and computational resources has opened up opportunities to calculate accessibility on a door-to-door level (Benenson et al. 2010; Lei and Church 2010) and to offer public transport travel accessibility calculation as a web service (OpenTripPlanner, Byrd 2012). OpenTripPlanner uses an open data format for public transport schedules and the openly available OpenStreetMap. OpenTripPlanner calculates the accessibility for a single point in time and single location. Results thus differ based on the chosen starting point and, more importantly, the chosen start time. Results are then dependent on the headway of the transit service. OpenTripPlanner has been applied to the Minneapolis/St. Paul region (Owen and Levinson 2015). By calculating the cumulative opportunity transit accessibility to jobs for different travel time bands, and for every minute in the morning peak, an average and maximum accessibility is calculated. The underlying pedestrian network is based on OpenStreetMap; the connection between destinations and the road network is not readily defined.

\section{Methodology, study region and data}

\subsection{Methodology}

In this study, we calculate accessibility with door-to-door travel times and thus account for every stage in a journey. If the four components of accessibility identified by Geurs and van Wee (2004) are translated to the building level, this leads to the following challenges: (1) Land-use: The measurement of opportunities on a building-fine level, (2) Transport: The incorporation of non-motorized modes and 
transit and the connection of the transport network to opportunities, (3) Temporal: Measuring transportation system performance and the availability of opportunities by time of day and (4) Individual: Taking into account the abilities of individuals to participate in the (non-motorized) transport system and the attractiveness of different opportunities to different individuals.

\subsection{Study region}

As a case study for this study we consider the city state of Singapore. Singapore is located in Southeast Asia with a land area of $712 \mathrm{~km}^{2}$, a permanent residential population of 3.77 million, and a total population of 5.08 million in 2010, compared to $697 \mathrm{~km}^{2}, 3.27$ million, and 4.03 million in 2000, respectively. GDP per capita amounts to $\$ \$ 59,813$ (US\$45,200, 2010), which makes it one of the wealthiest countries in (Southeast) Asia. Together with the increasing population and wealth, vehicle ownership has increased from 392,961 in 2000 to 597,746 in 2010, or from one car per 10 households in 2004 to one car per 8.8 households in 2008 (Choi and Toh 2010). The total number of motorized vehicles is close to one million. While the car has long been the mode of choice, the combination of an increasing population and a limited amount of certificates of entitlement to vehicles being available for auction, vehicle ownership has become a financially unattractive option for many households. Both the Singapore Land Transport Authority and the Urban Redevelopment Authority are seeking ways to make public transport more attractive on one hand and to bring jobs closer to residences on other hand.

\subsection{Available data}

\subsubsection{Opportunities: jobs}

Building fine opportunities are available for several activity types. In this study, we consider the the number of jobs per building. Chakirov and Erath (2012) have identified workplaces in Singapore by analyzing public transport smart card data; these workplaces have been inflated by mode share per TAZ as observed in the Household Interview Travel Survey (2008). Subsequently, Ordóñez Medina and Erath (2013) distributed public transport trips identified as commuting journeys to individual buildings by means of an iterative proportional fitting. The entire calculation environment is in accordance object-oriented design principles. In this paper, we present accessibility to jobs. However, in each building object additional attributes are stored, such as the number of residential units, land-use type, and several points of interest.

\subsubsection{Non-motorized transport: the case of the pedestrian}

In this study, we compare two pedestrian networks with a base scenario in which Euclidean distances are considered. Before proceeding to the differences between the two pedestrian networks, the commonalities will be highlighted. As a basis for both networks, the 2008 road center lines of the Singapore Land Authority (SLA) are used. This network has been chosen because the network coverage is comprehensive and the links are organized by road category. Expressways are considered as not available to pedestrians and are thus excluded from both networks.

Building footprints and accompanying addresses are available for 2008 from the SLA. These footprints are matched to road center lines by means of the shortest line between building centroid and roads with a name corresponding to the address of the building; each address can only have one line connected to the network.

Figure 1 gives an overview of the data sources used for the generation of both pedestrian networks.

Two pedestrian networks are generated. The first network consists of walkways on road center lines - a fairly traditional approach. It is assumed that each link is a sidewalk and dedicated crossings are not necessary. A building located on one side of a road can be directly accessed by a building located on the opposite side of the same road. Pedestrian overhead bridges and underpasses are added to this 
network if they intersect two or more road center lines. Bus stops, obtained from the Land Transport Authority (LTA), are matched to the road by means of shortest line. Mass Rapid Transit (MRT) stations are obtained from the LTA as well. Entrances and exits to these stations were downloaded from an online directory (Streetdirectory 2011) and matched to MRT stations by name. These entrance and exits are connected to the road center lines by means of the shortest lines. MRT entrances and exits are connected to the MRT station.

In addition to the road center lines, a number of additional data sources are used in the generation of the second pedestrian network-the "offset" pedestrian network. This network generation approach is inspired by the work of Parker and Vanderslice (2011) and Kim et al. (2009). Both studies propose methods for pedestrian network generation using center lines as a basis; however, their focus is not so much on creating a routable pedestrian network but to highlight possible sidewalk generation approaches.

In the road network, a distinction is made between nine road categories. Five road categories are assumed to have sidewalks on both sides of the road-a common feature in Singapore. For one category, the center line is used. The decision about whether a certain road category has a sidewalk on both sides of the road or whether the road center line is a walkway was made by sampling roads and inspecting them with Google Streetview. Pedestrian crossings are sourced from several data sets. Pedestrian overhead bridges and pedestrian underpasses are added to the offset road network. Pedestrian traffic lights are available but are not coded in pairs, making the matching of the traffic lights to the pedestrian network difficult; therefore, these are currently not included in the network. Lane markings indicating a pedestrian crossing are added where lane markings intersect road center lines. Lane markings not crossing a road center line are evaluated based on their distance to road center lines and the angle between the road center line and the lane marking. Pedestrians are always required to use a crossing. It should be noted that certain road categories are hard to cross mid-block due to the large number of lanes or fences placed along sidewalks (one-way roads) or in the middle of the road (two-way roads). One road category has the possibility of being crossed in some cases mid-block; this is not considered in the current network.

By following this approach, we create a network that provides a fixed and formal description of the network. In literature other methods can be found for the modeling of pedestrian movement, such as agent-based methods. They offer more flexibility in the types of input data required (e.g., Kukla et al. 2003; Penn and Turner 2001). Such methods would, for instance, allow for mid-block crossing and the crossing of squares. However, a pedestrian network offers better computational performance. More importantly, the question is whether one wants to account for mid-block crossings and jaywalking for planning purposes.

The Singapore climate and walking environment contains elements that could be considered discouraging for walking, such as a high humidity, overhead bridges, and underpasses. While the offset pedestrian network distances can approximate distances along roads, perceived costs at grade crossings, overhead bridges, and underpasses are underestimated since the number of lanes being crossed is not yet taken into account. The same is true for waiting times at signalized intersections-which can amount to 150 seconds and, in the case of large roads, twice this amount-since it is necessary to wait a second time halfway through the crossing. Furthermore, generalized costs for these types of crossings are not yet considered, nor are generalized costs regarding the sidewalks. These costs are discarded since no information is known about the relative weighing of attributes. Finally, the limitation of only one link between each building and the network is known not to represent reality in all cases. Social housing, shopping malls and parks are usually connected to more than one link in the network since effort has been made to make these environments pedestrian friendly. However, taking these limitations into account, the generated pedestrian networks offer the opportunity of connecting the public transport system to individual activity opportunities, thus connecting the macro transport system to the micro level. 


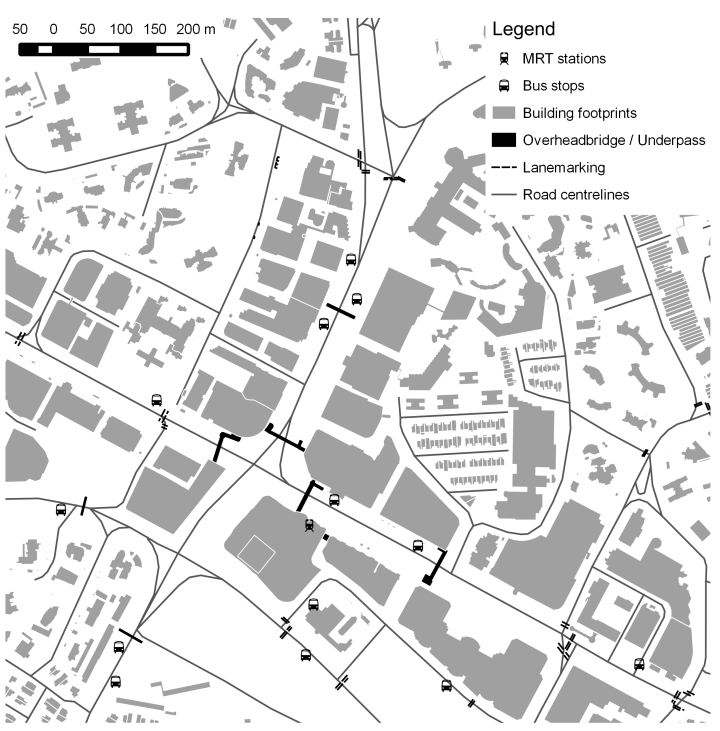

Figure 1: Source data

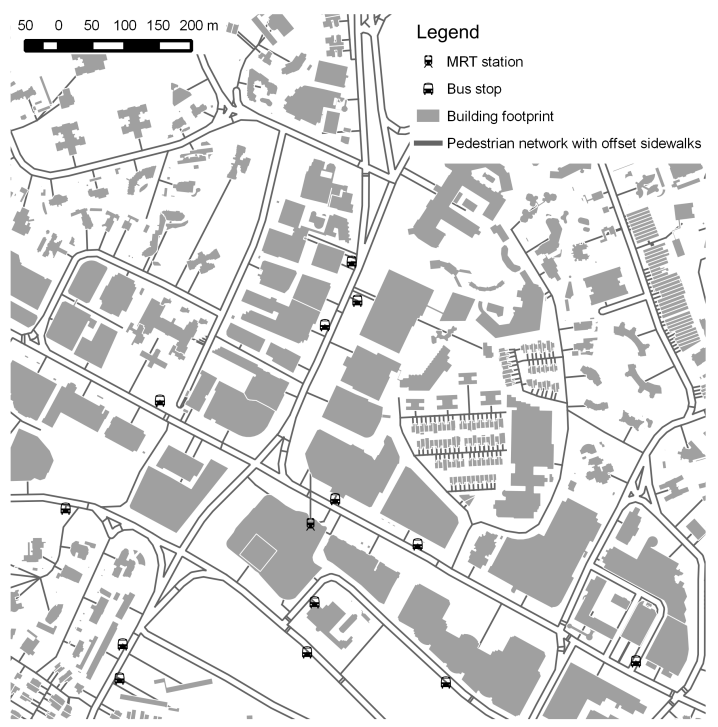

Figure 2: Pedestrian network (offset)

\subsubsection{Public transport travel times}

Public transport travel times are calculated with an implementation of a multi-agent, activity-based (MATSim) transport demand model of Singapore (Erath et al. 2012). The implementation of MATSim used in this study simulates smart card data, in which more than 4500 bus stops, 80 Mass Rapid Transit (MRT) stops, and 30 Light Rapid Transit (LRT) stops are included. Twenty-five percent of the daily 4.5 million public transport trips are simulated. As a simulation of only the public transport system would lead to unrealistically low travel times because no interaction with other modes takes place, link travel times and bus stop dwell times are estimated by using time stamps recorded in smart card data. The result of the iterative simulation is a temporally dynamic network with the average travel time for each link per 15-minute interval over the 24-hour time span. Subsequently, all possible transit stop combinations are routed on this loaded network. The shortest path algorithm returns the shortest transit time in each 15-minute interval, including transfer time and transfer walking time if applicable. The routing algorithm considers different taste parameters for in-vehicle time, transfer waiting time, and transfer walking time. These parameters are calibrated for the Singapore MATSim model.

\subsubsection{Execution of the accessibility calculation}

The calculation of building-fine accessibility indicators is split into a number of steps for both computation and validation purposes. All computations have been carried out in a multi-threaded environment on a HPC using a combination of JAVA, the Java Topology Suite (JTS), Postgres, and PostGIS. The results of each step are stored for further analysis. This section will discuss the steps carried out and the respective parameter settings in each step.

\subsubsection{Transit travel times}

In this study, we consider the period between 7:00 a.m. and 9:30 a.m. of an average weekday, corresponding to the morning peak hour. For each 15-minute interval in this period, the transit time from each transit stop to each other transit stop is available; the median of these travel times is used in further accessibility calculations. 


\subsubsection{Selection of candidate transit stops}

Euclidean distance and network distances between buildings and transit stops within a radius of 1000 meters of each building centroid are calculated. Out of the 113,406 buildings considered, 112,040 buildings have a transit stop within a 1000-meter radius, 108,164 buildings have a transit stop within a 1000-meter network distance considering a simple pedestrian network, and 106,850 buildings have a transit stop within a 1000-meter offset network distance. Figure 3 shows the number of buildings that have a transit stop within a certain distance; colors indicate the type of transit. More than 86 percent of the buildings have a transit stop within 300 meters if Euclidean distances are considered. If simple network distances are considered, this value drops to 52 percent. If the offset network is considered, 47 percent of the buildings have a transit stop within 300 meters.

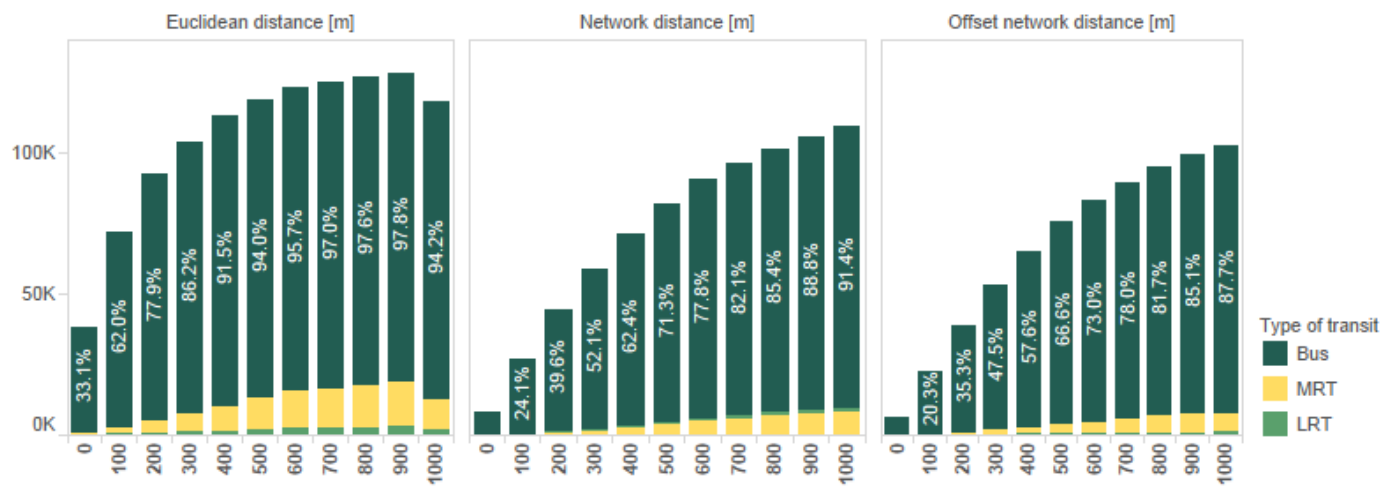

Figure 3: Number of buildings and distance to transit stop per network type

On average, each building is connected to 39 transit stops within a 1000-meter Euclidean distance. The average number of transit stops drops to 16 (1000-meter network distance) and 13 (1000-meter offset network distance).

\subsubsection{Calculate pedestrian accessibility}

Pedestrian accessibility is calculated by calculating the Euclidean distance and network distances between each building and all other buildings within a 1000-meter radius or 1000-meter network distance respectively. Calculated distances are converted to a travel time, assuming a walking speed of 4 $\mathrm{km} / \mathrm{h}$. Currently, the accessibility computation and accompanying distance decay function are implemented:

$$
A_{i}=\sum_{j=1}^{n} d_{j} \exp \left(\alpha t_{i j}\right)
$$

where $A_{i}$ is the total accessibility of building $i, d_{j}$ are the opportunities at destination $j, \alpha$ is the distance decay (impedance) parameter and $t_{i j}$ is the total travel time between $i$ and $j$. Job and residential opportunities are discounted, assuming an impedance factor of -0.2 for job opportunities as well as for residential opportunities. This impedance factor is based on survey data (Erath et al. 2012). Opportunities reached by walking are subsequently excluded from the total set of opportunities.

\subsubsection{Calculation of transit accessibility}

The travel time between each origin building, candidate transit stops for this building, and the transit travel time between each transit stop is calculated in the previous steps. In the final step, the options 


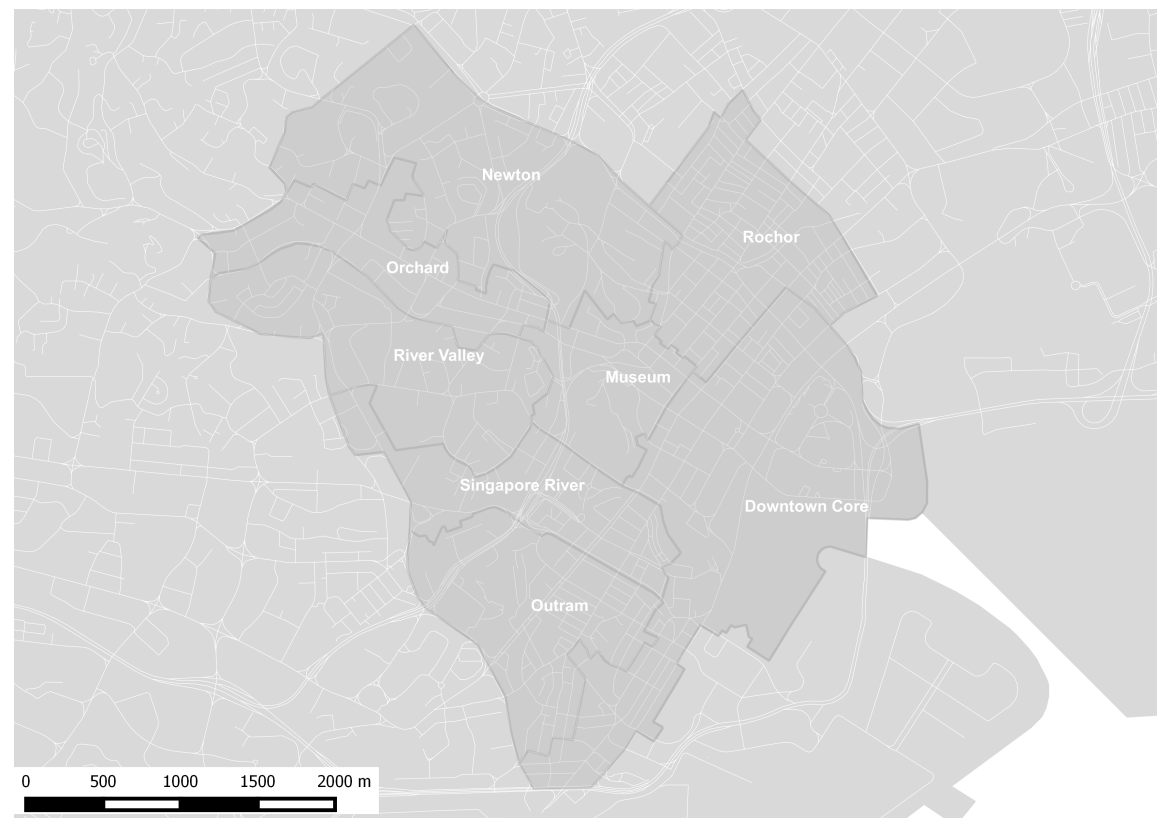

Figure 4: Selected areas highlighted in dark grey; white lines represent the road network.

between each destination building and its candidate stops are evaluated. Again, Equation 1 is used to calculate the accessibility of a building. The route with the shortest travel time, consisting of the walking stage from the origin, transit time, and the walking stage at the destination end of the trip, is selected for the accessibility calculation. Again, a walking speed of $4 \mathrm{~km} / \mathrm{h}$ is assumed. Furthermore, only transit stops within a 700-meter (Euclidean and network) distance are selected as candidate transit stops. This parameter is currently used for analysis purposes of the algorithm; in the future, this parameter will be replaced by a maximum distance for a bus stop and and a maximum distance for a MRT stop. These parameters will be based on the Household Interview Travel Survey.

\subsubsection{Calculation of total accessibility}

In the previous steps, walking distances have been converted to travel times in minutes. Transit times have also been converted to minutes. Total job accessibility for a single building is calculated as the sum of transit accessibility and pedestrian accessibility.

\section{Results}

This section presents the results of accessibility calculation. All transit accessibility measures include all possible buildings as destinations in Singapore. Computing pedestrian accessibility for buildings within a 1000-meter radius is a matter of seconds; calculating transit accessibility is highly dependent on the number of alternative routes to be evaluated and takes longer. Overall, the algorithm is robust but heavily dependent on the quality of the input data. This will be highlighted later in this section.

In this paper, we present the results of a number of selected zones. These zones are depicted in Figure 4 and consist of the main shopping belt Orchard Road starting in the west and sloping down to the east, as well as the Downtown Core and Museum District at the east end of Orchard Road. Orchard is considered to be the prime shopping district in Singapore. Its main road, Orchard Road, is a five-lane, one-way road. Crossing is only possible at a limited number of underpasses and grade crossings. Newton is a residential district situated on the north side of Orchard Road; River Valley is the residential area south of Orchard Road. Singapore River, Outram, and Rochor contain heritage 
Table 1: Selected planning zones

\begin{tabular}{lrrr}
\hline Planning zone & \# Buildings & Estimated work place capacity & \# Residential units \\
\hline Downtown Core & 413 & 161757 & 1762 \\
Museum & 111 & 15259 & 624 \\
Newton & 776 & 5335 & 7402 \\
Orchard & 145 & 54248 & 1488 \\
Outram & 1472 & 26633 & 7666 \\
River Valley & 761 & 1396 & 7691 \\
Rochor & 2256 & 24284 & 5072 \\
Singapore River & 438 & 19294 & 4615 \\
\hline
\end{tabular}

quarters and have a fine-grained street pattern. Table 1 presents key indicators of the selected zones; the Downtown Core and Orchard have the majority of jobs while the other zones are mainly residential.

Table 2: Selected planning zones and average pedestrian and transit accessibility per building

\begin{tabular}{|c|c|c|c|c|c|c|}
\hline Planning zone & $\begin{array}{l}\text { Pedestrian } \\
\text { accessibil- } \\
\text { ity to jobs } \\
\text { (Euclidean) }\end{array}$ & $\begin{array}{l}\text { Pedestrian } \\
\text { accessibil- } \\
\text { ity to jobs } \\
\text { (Centreline) }\end{array}$ & $\begin{array}{l}\text { Pedestrian } \\
\text { accessibility to } \\
\text { jobs (Offset) }\end{array}$ & $\begin{array}{l}\text { Transit acces- } \\
\text { sibility to jobs } \\
\text { (Euclidean) }\end{array}$ & $\begin{array}{l}\text { Transit acces- } \\
\text { sibility to jobs } \\
\text { (Centreline) }\end{array}$ & $\begin{array}{l}\text { Transit acces- } \\
\text { sibility to jobs } \\
\text { (Offset) }\end{array}$ \\
\hline Downtown Core & 27182 & 11583 & 8152 & 43564 & 21936 & 20210 \\
\hline Museum & 13764 & 4104 & 3536 & 51319 & 24075 & 21455 \\
\hline Newton & 11242 & 2791 & 1922 & 33290 & 10048 & 8671 \\
\hline Orchard & 15566 & 6484 & 4741 & 51120 & 24137 & 21503 \\
\hline Outram & 23047 & 8266 & 5466 & 31803 & 15356 & 13620 \\
\hline River Valley & 8844 & 1847 & 1361 & 27503 & 12726 & 11048 \\
\hline Rochor & 10222 & 4148 & 3488 & 34424 & 17042 & 14440 \\
\hline Singapore River & 25170 & 10890 & 3954 & 40254 & 18314 & 11174 \\
\hline
\end{tabular}

\subsection{Results on a zonal level}

In Table 2, the average accessibility per building per planning zone is shown. For both pedestrian and transit accessibility, a drop can be observed between computations using Euclidean distances between opportunities and network distances. In the Downtown Core, Rochor, and Singapore River, the smallest decreases can be observed. This can reflect the dense street pattern in these zones. Differences in accessibility between the center line and offset network for pedestrian accessibility can also be observed, albeit smaller than as compared to Euclidean distances. The Downtown Core is well connected by underpasses and grade crossings; the Singapore River and Rochor zones contain streets that can be crossed at any point. A second reason for the small difference between the results of both pedestrian networks can be that the length of grade crossings, overhead bridges, and underpasses is limited in this study to 5 meters. The drop between Euclidean and network-based pedestrian accessibility is much steeper in the Museum, Newton, and River Valley planning zones. The last two planning zones have a large number of condominiums and a low number of crossings, leading to a low pedestrian accessibility. The drop in transit accessibility is also visible but less steep. This indicates that a low number of job opportunities is available in these zones, and motorized transport is required to reach job opportunities.

\subsection{Results on a building level}

Figures 5, 6, and 7 place the results in a spatial context. The top figure shows accessibility for the case when public transport is connected to buildings by means of Euclidean distance; the middle figures show accessibility when a road center line network is used for walking distances. The bottom figure 
shows accessibility when offset network distances are considered. Legends are explicitly chosen to be in the same range to allow for direct comparison.

It can be seen that buildings near transit stops have a high job accessibility in the Downtown Core and Orchard Road. The Newton zone to the top and the River Valley zone below have a low transit accessibility, with the accessibility values dropping more quickly in the case of center line and offset distances, as expected. The differences between center line and offset-based accessibility are less apparent from Figure 6 and Figure 7. Differences can be found in accessibility when comparing buildings on both sides of major roads. This is probably because of the detour required to reach transit stops in the case of the offset network.

Figure 8 shows the relative difference between Euclidean and network-based transit accessibility per building. Differences in accessibility are smallest for buildings near transit stops-an intuitive result. Also, differences are smaller in areas with smaller buildings and finer-grained street patterns. Examples are the Little India district of the Rochor planning zone in the top right and the China Town district in the Outram/Singapore River planning zone in the bottom right.

This visual comparison also highlights the challenges when computing accessibility on the microlevel. Figure 5 shows high accessibility for the area in the far right, indicated by the letter M. Accessibility when considering road center line distance for this area are lower, while accessibility values when considering the offset network distances drop even further. Closer inspection tells us that there is a limited number of grade crossings in this area; this is reflected in the offset pedestrian network. However, in reality the area contains large shopping malls that can be traversed, as well as an underground mall. These pathways are not yet included in the pedestrian model. However, research is required if these mall are accessible 24 hours per day to pedestrians, and if these indoor pathways are known to pedestrians. The second case concerns Raffles Places, demarcated by an $\mathrm{R}$ in the bottom of all accessibility maps. Raffles Place is one of the main centers of the Downtown Core and is well-connected by the two main MRT lines. Closer inspection in this case reveals that buildings in this area have Raffles Place as their address, but Raffles Place as a street is not included in the road network since it is a carfree, pedestrian-only square. Near Raffles Place, a smaller street block is not connected to the larger network. In this, closer inspection shows us that, based on the road category, crossings are required; the official source data, however, does not contain these crossings. These cases highlight the need for highly detailed data and but also for the need for comparison with ground-truth.

\section{Conclusions and remaining challenges}

\subsection{Conclusions}

This paper has presented a combined pedestrian and transit accessibility measure and quantified this measure for the central area of Singapore. Transit stops have been connected to a pedestrian network; for train stations, an additional effort has been made to include entrances and exits. It has been shown that job accessibility strongly decreases if, instead of Euclidean distance to transit stops, network distances are considered. Differences in accessibility measures, considering different pedestrian distance estimates, are smaller in areas with a dense street pattern and smaller parcel sizes. The accessibility of buildings near a transit stop indicates how well jobs can be reached from this transit stop-an important factor when considering the quality of public transport on a larger scale. Other types of activity opportunities are not yet in included in the calculations. Results are dependent on the quality of the input data. A pedestrian network based on offset center lines requires additional data sources to include pedestrian crossings. The fact that this data is not readily available might indicate that transport and land-use authorities currently do not have the tools necessary to analyze pedestrian accessibility. For certain types of buildings (shopping malls, condominiums, public housing), a closer look is necessary at the connection of buildings and parcels to the network. Accessibility calculations do not differ much between the two types of pedestrian networks. On one hand, this is a comforting result, as it 


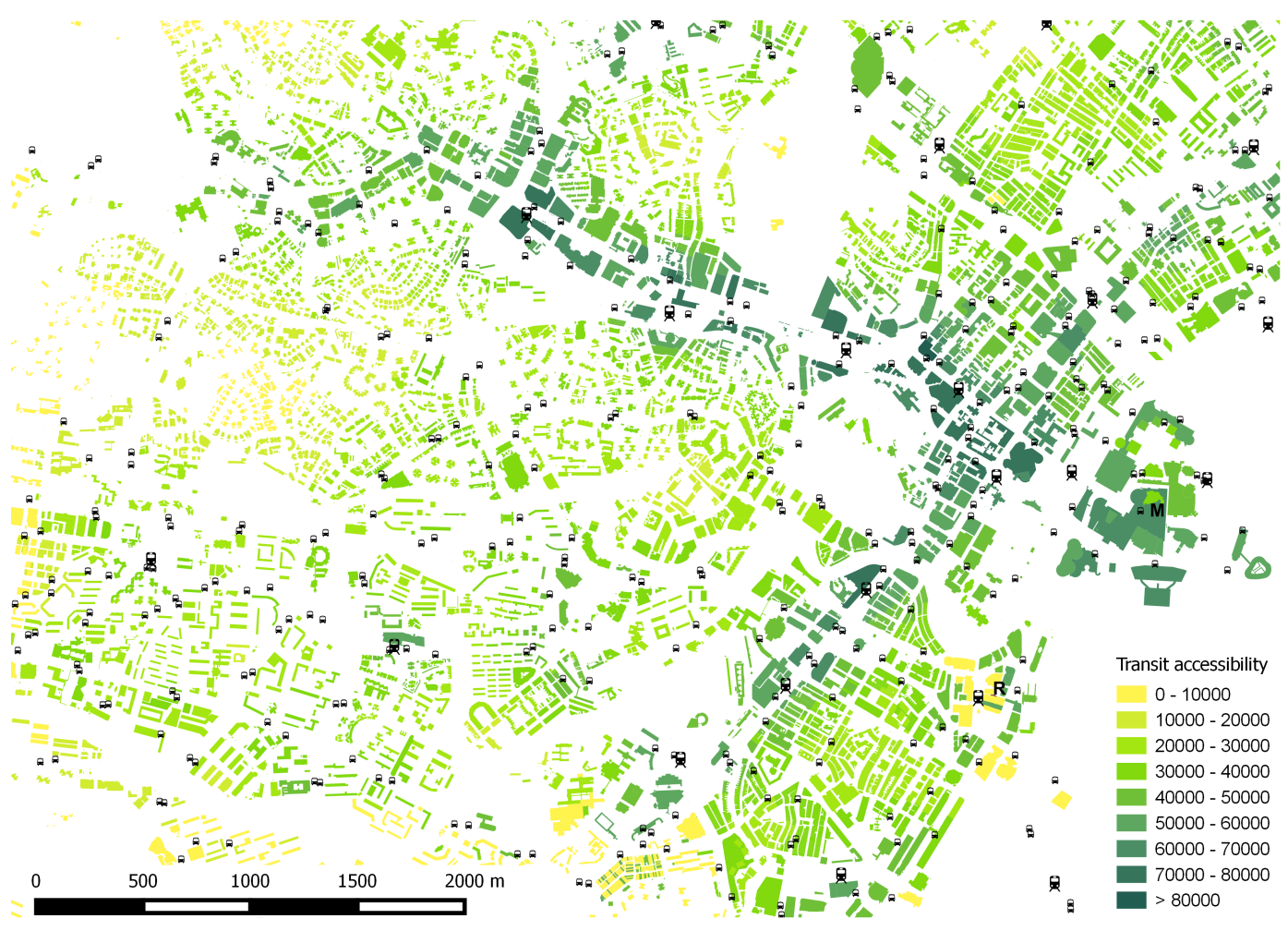

Figure 5: Transit accessibility to jobs, Euclidean distances to transit.

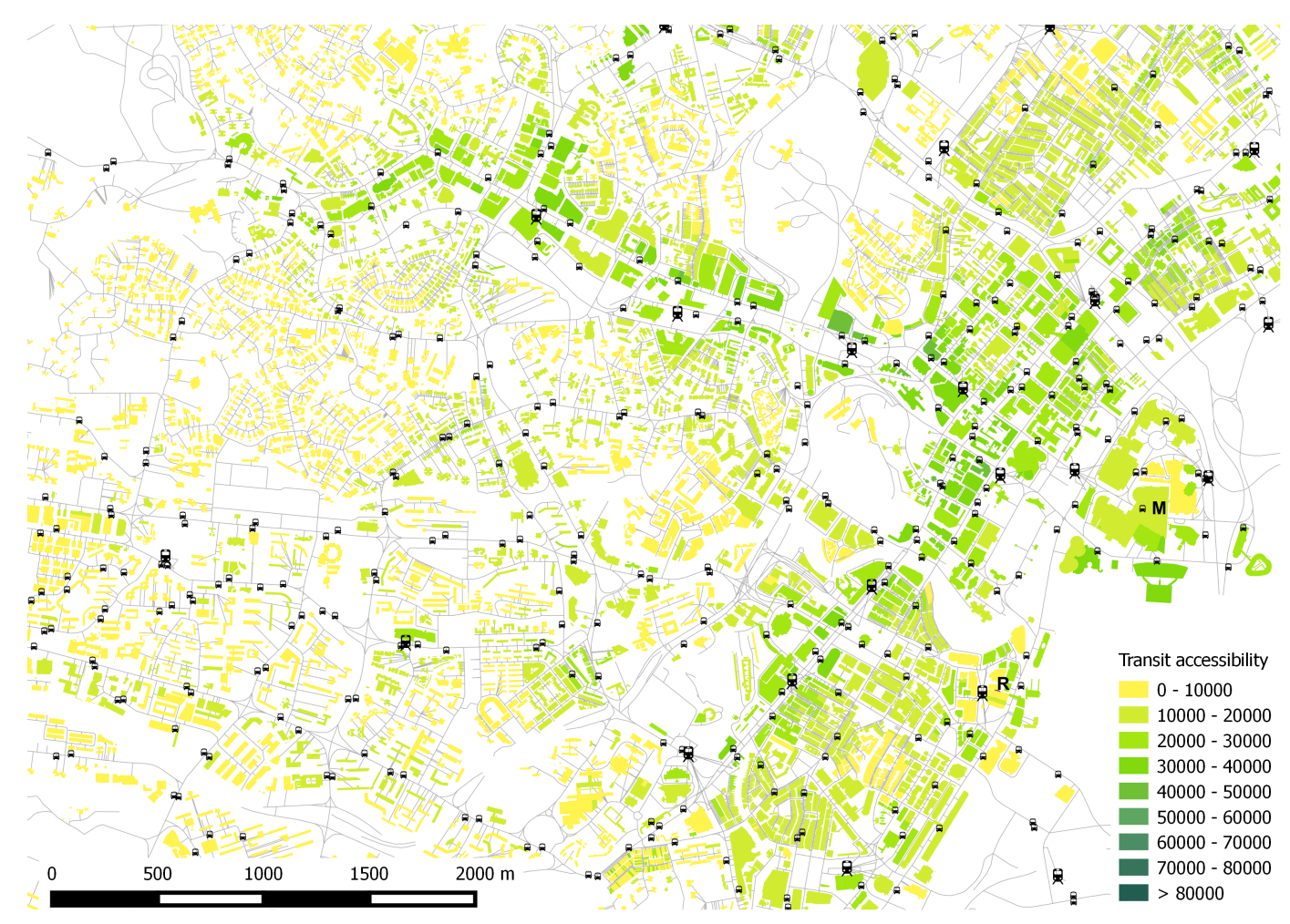

Figure 6: Transit accessibility to jobs, center-line pedestrian network. 


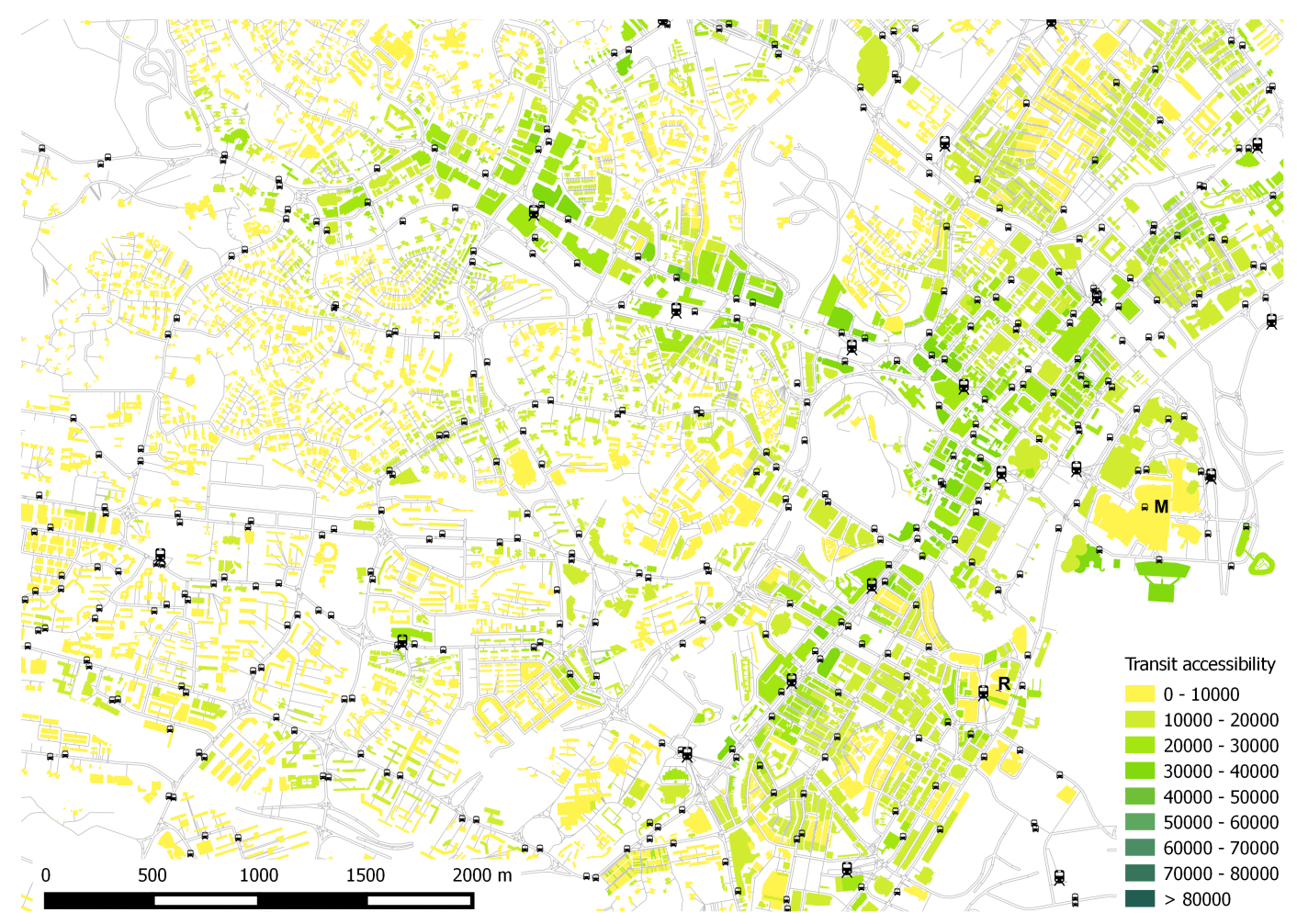

Figure 7: Transit accessibility to jobs, offset pedestrian network.

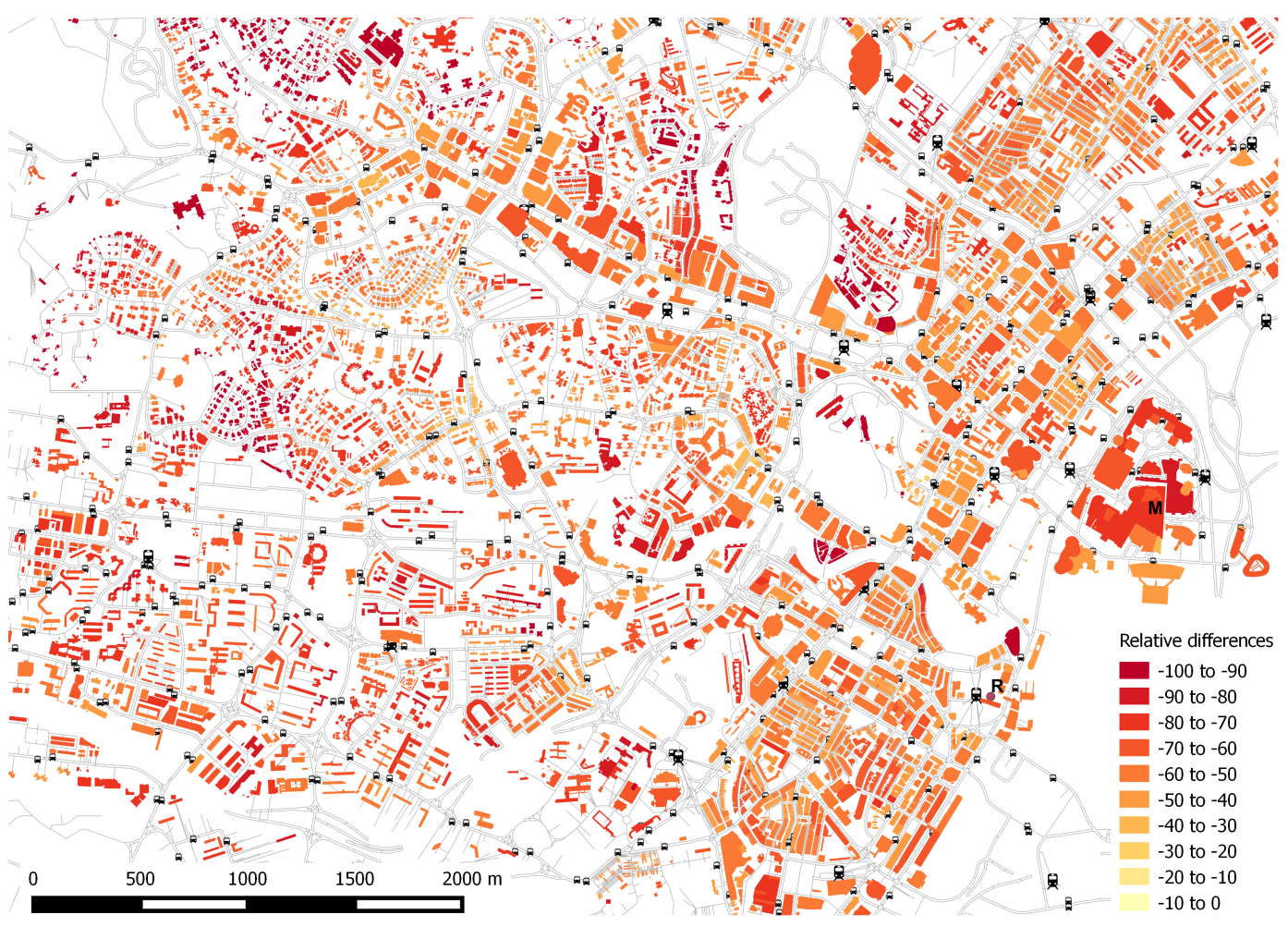

Figure 8: Relative differences between Euclidean and network-based transit accessibility per building. 
indicates that our offset pedestrian network is well-connected to points of interest in the network. On other hand, this result might be seen as surprising; extra analysis is required to compare the generalized costs of routes instead of distances between the two networks.

\subsection{Remaining challenges}

\subsubsection{Classifying opportunities, destination similarity, and destination competition}

While data is available concerning shopping malls, food centers, coffee shops, schools, supermarkets, and a wide range of other opportunities, it is the abundance of data and the necessary categorization of the data that concerns us. Therefore, we plan to investigate the valuation of similar activity opportunities in the destination set. This similarity investigation will include a directional component-i.e., are amenities in the same direction or at the same location valued more or less than amenities spread out through space? Furthermore, when measured on the local scale, it is expected that amenity categories have to be reformulated; individual A might prefer coffee $S$ and the price level of supermarket $\mathrm{Y}$, while individual $\mathrm{B}$ can prefer coffee $\mathrm{T}$ and the price level of supermarket $\mathrm{Z}$ and thus value coffee shops and supermarkets differently than individual A. However, does this create the necessity to create a category for each type of coffee shop and each supermarket brand? On other hand, accessibility is about reaching destinations. It can also be argued that simply reaching more destinations, and having more choice, is beneficial.

\subsubsection{Pedestrian network generation with open source data and generalized link costs}

A second challenges lies in the generation of pedestrian networks, the assignment of general costs to the links, and the generation of different networks for different user groups. The network generation approach followed is heavily dependent on propitiatory data sources and different sets of rules for different crossing types, but it can easily be changed to open data (e.g., OpenStreetMap). Surprisingly, although the data sources used in this study contain a tremendous amount of information of relevance to a pedestrian network, the information stored is not suitable for pedestrian network generation and the analysis of non-motorized accessibility, as previously recognized in literature (Handy and Clifton 2001a; Iacono et al. 2013). When considering accessibility on a micro level, the modeling of connection between buildings and the network become important; an approach solely based on a connection between buildings and roads with the same road name, especially in shopping malls and Singaporean housing estates that have extensive pedestrian walkways, is not sufficient. However, simply connecting buildings to the nearest links might also not be representative of reality. First steps to include housing estates, parks, and malls in a network combined with gridcells have been made (van Eggermond and Erath 2013). Public transport travel times are currently based on the results of an agent-based simulation. However, for a large amount of cities, public transport schedules are available in the form of the General Transit Feed Specification (GTFS). While GTFS only represents the transit schedule and not the real-world execution, data are available for a wide range of cities and regions.

\subsubsection{Parameter settings and accessibility algorithm}

A third challenge can be found in setting of the different parameters, such as destinations that can be reached by walking and the maximum distance to transit stops. Walking distances can be extracted from household travel surveys; a maximum walking distance for destinations to be considered accessible by foot is harder to estimate since it is highly likely that non-motorized trips are underreported. Calculation time is very much dependent on the number of options to be evaluated. While the accessibility calculation for a single building is still acceptable (taking only a matter of seconds), computations for entire districts take much longer. Decreasing the number of transit stop and building combinations will decrease the number of origin and destination travel alternatives to be evaluated; especially in the city center, a large amount of transit stops are included in calculations. A second option is to decrease 
the number of destinations to be evaluated, either by setting a cut-off time or by considering only buildings with certain opportunity types. Finally, there is the possibility of implementing a distance decay function suitable for short distances (Martínez and Viegas 2013).

\subsubsection{Interactive visualization and application}

The current visualizations are static; all results are pre-computed and stored in a database. In line with the possibilities of OpenTripPlanner Analyst (Byrd 2012), it is envisaged that users can interact with different parameter settings and take the possibilities a step further by offering users the ability to change the pedestrian network and evaluate the changes in accessibility. By following this approach, we hope that it is possible to truly connect the distant to the local and support local planners and citizens with tools to assess and improve accessibility.

\section{Acknowledgements}

The research conducted at the Future Cities Laboratory is co-funded by the Singaporean National Research Fund and the ETH Zurich. The authors would like to thank Professor Kay W. Axhausen for his suggestions and comments. Also, we would like to thank Sergio Ordonez Medina for his help calculating transit travel times. We wish to express our gratitude to the Land Transport Authority for providing us with invaluable data sets on transport in Singapore. We are very thankful to the Singapore Land Authority for providing us with a wide range of data sets. 


\section{References}

Benenson, I., K. Martens, Y. Rofé, and A. Kwartler. 2010. Public transport versus private car gisbased estimation of accessibility applied to the tel aviv metropolitan area. Annals of Regional Science, 47(3):499-515.

Bhat, C. R., S. L. Handy, K. Kockelman, H. S. Mahmassani, A. Gopal, I. M. Srour, and L. Weston. 2002. Development of an Urban Accessibility Index: Formulations, Aggregation, and Application. Working Paper Report 7-4938-4, University of Texas Austin, Austin.

Bundesamt für Raumentwicklung ARE. 2011. ÖV-Güteklassen - Berechnungsmethodik ARE Grundlagenberichten. Technical report, Swiss Federal Office for Spatial Development (ARE), Berne.

Byrd, A. 2012. Visualizing urban accessibility with OpenTripPlanner Analyst. webpage.

Chakirov, A. and A. Erath. 2012. Activity identification and primary location modelling based on smart card payment data for public transport. In IATBR, ed., 13th International Conference on Travel Behaviour Research (IATBR). Toronto.

Choi, C. C. and R. Toh. 2010. Household interview surveys from 1997 to 2008: A decade of changing travel behaviours. Journeys, 5(May 2010):52-61.

Erath, A., P. J. Fourie, M. A. B. van Eggermond, S. A. Ordóñez Medina, A. Chakirov, and K. W. Axhausen. 2012. Large-scale agent-based transport travel demand model for Singapore. In IATBR, ed., 13th International Conference on Travel Behaviour Research (IATBR). Toronto.

Geurs, K. T. and B. van Wee. 2004. Accessibility evaluation of land-use and transport strategies: review and research directions. Journal of Transport Geography, 12(2):127-140.

Hägerstrand, T. 1970. What about people in regional science? Papers of the Regional Science Association, 24(1):7-21.

Handy, S. L. and K. J. Clifton. 2001a. Evaluating neighborhood accessibility: Possibilities and practicalities. Journal of Transportation and Statistics, 4:67-78.

Handy, S. L. and K. J. Clifton. 2001b. Local shopping as a strategy for reducing automobile travel. Transportation, 28(4):317-346.

Hansen, W. 1959. How Accessibility Shapes Land Use. Journal of the American Institute of Planners, 25(2):73-76.

Hess, S. 2005. Access to employment for adults in poverty in the buffalo-niagara region. Urban Studies, 42(7):1177-1200.

Iacono, M., K. J. Krizek, and A. El-Geneidy. 2013. Measuring non-motorized accessibility: issues, alternatives, and execution. Journal of Transport Geography, 18:133-140.

Karimi, H. A. and P. Kasemsuppakorn. 2012. Pedestrian network map generation approaches and recommendation. International Journal of Geographical Information Science, 27(5):1-16.

Kawabata, M. 1998. User-equilibrium properties of fixed points in dynamic traffic assignment. Transportation Research Part C: Emerging Technologies, 41(1):183-198.

Kim, J., S. y. Park, Y. Bang, and K. Yu. 2009. Automatic derivation of a pedestrian network based on existing spatial data sets. In ASPRS/MAPPS 2009 Fall Conference. San Antonio.

Kukla, R., A. Willis, and J. Kerridge. 2003. Application of context-mediated behavior to a multi-agent pedestrian flow model (PEDFLOW). In TRB, ed., 82nd Annual Meeting of the Transportation Research Board. Washington, D.C.: Transportation Research Board.

Lei, T. L. and R. L. Church. 2010. Mapping transit-based access: integrating GIS, routes and schedules. International Journal of Geographical Information Science, 24(2):283-304.

Levinson, D. 1998. Accessibility and the journey to work. Journal of Transport Geography, 6(1):11-21. Martínez, F. and J. M. Viegas. 2013. A new approach to modelling distance-decay functions for accessibility assessment in transport studies. Journal of Transport Geography, 26:87-96.

Ordóñez Medina, S. A. and A. Erath. 2013. Estimating dynamic workplace capacities by means of public transport smart card data and household travel survey in Singapore. Transportation Research 
Record, 2344:20-30.

Owen, A. and D. Levinson. 2015. Modeling the commute mode share of transit using continuous accessibility to jobs. Transportation Research Part A: Policy and Practice, 74:110-122.

Parker, J. S. and E. Vanderslice. 2011. Pedestrian network analysis. In XII International Walk21 Conference on Walking and Liveable Communities. Vancouver.

Penn, A. and A. Turner. 2001. Space syntax based agent simulation. In 1st International Conference on Pedestrian and Evacuation Dynamics. Duisburg.

Salonen, M. and T. Toivonen. 2013. Modelling travel time in urban networks: comparable measures for private car and public transport. Journal of Transport Geography, 31:143-153.

Shen, Q. 1998. Location characteristics of inner-city neighborhoods and employment accessibility of low-wage workers. Environment and Planning B, 25(3):345-365.

Speck, J. 2012. Walkable City: How Downtown Can Save America, One Step At A Time. Farrar Strauss \& Giroux.

Streetdirectory. 2011. Streetdirectory Singapore. webpage. Accessed on 1/04/2012.

van Eggermond, M. A. B. and A. Erath. 2013. Accessibility on a micro-level: a closer look at pedestrian routing and network generation. In EASTS, ed., 10th International Conference of Eastern Asia Society for Transportation Studies. Taipei. URL www.ivt.ethz.ch/vpl/publications/\#860.

Walk Score. 2014. Walk Score. webpage.

Weinstein Agrawal, A., M. Schlossberg, and K. Irvin. 2008. How Far, by Which Route and Why? A Spatial Analysis of Pedestrian Preference. Journal of Urban Design, 13(1):81-98. 